\title{
Front Matter: Volume 9164
}

, "Front Matter: Volume 9164," Proc. SPIE 9164, Optical Trapping and Optical Micromanipulation XI, 916401 (9 October 2014); doi: 10.1117/12.2086699

SPIE Event: SPIE NanoScience + Engineering, 2014, San Diego, California, United SPIE. States 




\section{Optical Trapping and Optical Micromanipulation XI}

Kishan Dholakia

Gabriel C. Spalding

Editors

17-21 August 2014

San Diego, California, United States

Sponsored and Published by

SPIE 
The papers included in this volume were part of the technical conference cited on the cover and title page. Papers were selected and subject to review by the editors and conference program committee. Some conference presentations may not be available for publication. The papers published in these proceedings reflect the work and thoughts of the authors and are published herein as submitted. The publisher is not responsible for the validity of the information or for any outcomes resulting from reliance thereon.

Please use the following format to cite material from this book:

Author(s), "Title of Paper," in Optical Trapping and Optical Micromanipulation XI, edited by Kishan Dholakia, Gabriel C. Spalding, Proceedings of SPIE Vol. 9164 (SPIE, Bellingham, WA, 2014) Article CID Number.

ISSN: 0277-786X

ISBN: 9781628411911

\section{Published by}

\section{SPIE}

P.O. Box 10, Bellingham, Washington 98227-0010 USA

Telephone +1 3606763290 (Pacific Time) · Fax +1 3606471445

SPIE.org

Copyright @ 2014, Society of Photo-Optical Instrumentation Engineers.

Copying of material in this book for internal or personal use, or for the internal or personal use of specific clients, beyond the fair use provisions granted by the U.S. Copyright Law is authorized by SPIE subject to payment of copying fees. The Transactional Reporting Service base fee for this volume is $\$ 18.00$ per article (or portion thereof), which should be paid directly to the Copyright Clearance Center (CCC), 222 Rosewood Drive, Danvers, MA 01923. Payment may also be made electronically through CCC Online at copyright.com. Other copying for republication, resale, advertising or promotion, or any form of systematic or multiple reproduction of any material in this book is prohibited except with permission in writing from the publisher. The CCC fee code is 0277-786X/14/\$18.00.

Printed in the United States of America.

Publication of record for individual papers is online in the SPIE Digital Library.

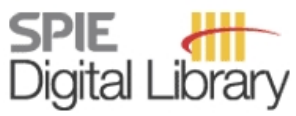

SPIEDigitalLibrary.org

Paper Numbering: Proceedings of SPIE follow an e-First publication model, with papers published first online and then in print and on CD-ROM. Papers are published as they are submitted and meet publication criteria. A unique, consistent, permanent citation identifier (CID) number is assigned to each article at the time of the first publication. Utilization of CIDs allows articles to be fully citable as soon as they are published online, and connects the same identifier to all online, print, and electronic versions of the publication. SPIE uses a six-digit CID article numbering system in which:

- The first four digits correspond to the SPIE volume number.

- The last two digits indicate publication order within the volume using a Base 36 numbering

system employing both numerals and letters. These two-number sets start with 00, 01, 02, 03, 04, 05, 06, 07, 08, 09, 0A, 0B ... 0Z, followed by 10-1Z, 20-2Z, etc.

The CID Number appears on each page of the manuscript. The complete citation is used on the first page, and an abbreviated version on subsequent pages. Numbers in the index correspond to the last two digits of the six-digit CID Number. 


\title{
Contents
}

\author{
ix Authors \\ xiii Conference Committee
}

TOWARD (OR IN) THE QUANTUM LIMIT OF OPTO-MECHANICS

916403 Cavity cooling a trapped nanosphere in vacuum [9164-2]

916404 Cooling the centre-of-mass motion of a silica microsphere [9164-3]

916405 Parametric stabilization and cooling of microparticles in a quadrupole ion trap [9164-4]

916407 On-chip optical trapping for atomic applications [9164-6]

\section{FOUNDATIONS OF THE ELECTROMAGNETIC THEORY OF FORCE AND MOMENTUM}

$91640 \mathrm{~A}$ Momentum measurements with holographic optical tweezers for exploring force detection capabilities on irregular samples [9164-9]

$9164 \mathrm{OB}$ Electromagnetic force and torque in Lorentz and Einstein-Laub formulations [9164-10]

\section{BEAM SHAPING AND ABERRATION/WAVEFRONT CORRECTION}

$91640 G$ Spontaneous revolution of micro-swimmers in a spherically aberrated optical trap [9164-92]

9164 0l Engineering particle trajectories in microfluidic flows using speckle light fields [9164-15]

\section{HELICITY AND OPTICAL ANGULAR MOMENTUM}

$91640 \mathrm{~J}$ Observation of the rotational Doppler effect from an optically trapped micro-particle [9164-16]

9164 OK Optical trapping with a perfect vortex beam [9164-17]

$9164 \mathrm{OL}$ Behavior of oblate spheroidal microparticles in a tightly focused optical vortex beam [9164-18]

$91640 \mathrm{M}$ Interesting manifestations of spin orbit interaction and spin Hall shift of light in an optical trap [9164-19] 
OPTICALLY DRIVEN MICRORHEOLOGY AND MECHANICAL PROPERTIES

916400 Measurement of interparticle force between nematic colloids [9164-21]

9164 OR Temporal response of biological cells to high-frequency optical jumping and vibrating tweezers [9164-26]

USING THE PHOTONIC TOOLBOX TO STUDY CELLS AND THEIR ORGANELLES

9164 OS Local probing and stimulation of neuronal cells by optical manipulation (Invited Paper) [9164-27]

9164 OU Force measurements with optical tweezers inside living cells [9164-29]

9164 OY The Pocketscope: a spatial light modulator based epi-fluorescence microscope for optogenetics [9164-33]

\section{ENHANCED SENSITIVITY AND RESOLUTION OF OPTICAL FORCE ACTUATORS}

916410 Quad stereo-microscopy [9164-35]

$916411 \quad$ Enabling accurate photodiode detection of multiple optical traps by spatial filtering [9164-36]

916412 A force measurement instrument for optical tweezers based on the detection of light momentum changes [9164-37]

916413 Optical tweezers escape forces [9164-38]

916414 A new technique for high sensitive detection of rotational motion in optical tweezers by a differential measurement of backscattered intensity [9164-39]

916415 Optical tweezers calibration with Bayesian inference (Invited Paper) [9164-40]

SINGLE-MOLECULE MANIPULATION AND STUDY

916418 Optical tweezers for free-solution label-free single biomolecule studies [9164-43]

PHOTONIC DEVICES FOR OPTICALLY INDUCED FORCES

$91641 \mathrm{E} \quad$ Inducing forced and auto oscillations in one-dimensional photonic crystals with light [9164-49] 
NEAR-FIELD MICROMANIPULATION, PLASMONIC, AND NANOPARTICLE TRAPPING

9164 IG Optical trapping of nanoscale plasmonic optical lattice in microfluidic environments [9164-51]

$91641 \mathrm{H}$ Promoting optofluidic actuation of microparticles with plasmonic nanoparticles [9164-52]

916411 Characterisation of Au nanorod dynamics in optical tweezers via localised surface plasmon resonance spectroscopy [9164-53]

9164 1L Anomalous dynamic behavior of optically trapped high aspect ratio nanowires [9164-56]

$91641 \mathrm{M}$ Optical trapping with pillar bowtie nanoantennas [9164-57]

\section{STUDIES OF ACTIVE SWIMMERS/HYDRODYNAMICS}

9164 iN Active matter transport on complex substrates [9164-58]

9164 IP Collective behavior of the optically driven particles on a circular path [9164-60]

$91641 Q \quad$ Investigating hydrodynamic synchronisation using holographic optical tweezers (Invited Paper) [9164-61]

\section{STATISTICAL MECHANICS OF SMALL SYSTEMS}

$91641 \mathrm{~V}$ Einstein's osmotic equilibrium of colloidal suspensions in conservative force fields (Invited Paper) [9164-67]

\section{OPTICAL MANIPULATION OF MATTER THROUGH GASEOUS MEDIA}

9164 IW Micro-rheology and interparticle interactions in aerosols probed with optical tweezers [9164-68]

$91641 \mathrm{X}$ The study of thin films on solid aerosol particles using optical trapping and Mie scattering from a broadband white LED [9164-69]

9164 1Y Aerosol optical chromatography and measurements of light extinction by single particles [9164-70]

OPTICAL SORTING, OPTICAL LAB-ON-A-CHIP, AND MICROFLUIDICS

916420 Ordering of colloids with competing interactions on quasi-one-dimensional periodic substrates [9164-73]

$916421 \quad$ Hybrid optical and acoustic force based sorting [9164-74]

916425 Optoelectronic cell lysis [9164-78] 
916426 Pulsed laser activated cell sorter (PLACS) for high-throughput fluorescent mammalian cell sorting [9164-79]

\section{OPTICALLY BOUND MATTER}

916429 Waveguides in colloidal nanosuspensions [9164-82]

\section{OPTOFLUIDICS AND OPTICALLY SHAPED STRUCTURES}

9164 2B Manipulation of particles by laser tweezers-induced gradient of order in the nematic liquid crystal [9164-84]

$91642 \mathrm{C}$ The break-up dynamics of liquid threads revealed by laser radiation pressure and optocapillarity [9164-85]

\section{OPTICALLY MANIPULATED ROBOTICS AND NOVEL SAMPLES}

$91642 \mathrm{~F}$ Optical tweezers as manufacturing and characterization tool in microfluidics [9164-88]

THE FINAL SESSION

916421 Optical trapping of isolated mammalian chromosomes [9164-123]

POSTER SESSION

9164 2L Micro-particles self-arrangement in shapeable counter-propagating beams [9164-96]

$91642 \mathrm{M} \quad$ Rapid fabrication of polymeric micro lenses for optical fiber trapping and beam shaping [9164-97]

$91642 \mathrm{~N} \quad$ Generation of shock waves in a medium with absorbing particles [9164-98]

916420 Viscoelasticity measurements inside liposomes [9164-99]

$91642 \mathrm{P}$ Natural user interface as a supplement of the holographic Raman tweezers [9164-100]

$91642 S$ Simulation of active Brownian particles in optical potentials [9164-103]

$91642 T \quad$ Multiplexed spectroscopy with holographic optical tweezers [9164-104]

$91642 \mathrm{U} \quad$ Numeric modeling approximation of the fluid dynamics in an optical fiber trap [9164-105]

$91642 \mathrm{~V}$ Holographic generation of vector beams with upper-bound diffraction efficiency [9164-106]

$91642 \mathrm{~W}$ Studying biofuel aerosol evaporation rates with single particle manipulation [9164-107] 
$91642 X \quad$ Optical nanofiber integrated into an optical tweezers for particle manipulation and in-situ fiber probing [9164-109]

$91642 \mathrm{Y}$ Towards polarization-sensitive trapping of nanoparticles in nanoring apertures [9164-110]

$91642 Z$ Multiscale manipulation of microbubbles employing simultaneous optical and acoustical trapping [9164-111]

916430 Micro- and nano-particle trapping using fibered optical nano-tweezers [9164-112]

916433 Holographic optical tweezers: microassembling of shape-complementary 2PP building blocks [9164-115]

916434 Tunable WGM resonators from optically trapped dye doped liquid crystal emulsion droplets [9164-116]

916435 Force dependence of phagosome trafficking in retinal pigment epithelial cells [9164-117]

916436 Anomalous behavior of a three-dimensionally optically trapped super-paramagnetic particle [9164-1 18]

916439 Beam-splitting waveguides induced in nanocolloids [9164-122]

9164 3A Generation of hollow optical beams for optical manipulation [9164-124]

9164 3B Plasmonic Archimedes spiral for selective optical trapping and rotation of optically isotropic particles [9164-125]

$91643 \mathrm{C}$ Trapping and manipulation of microparticles using Rayleigh convection generated by laser-induced heating of an absorbing thin film [9164-126]

9164 3D Various superpositions of Bessel beams for capture and controlled rotation of microobjects [9164-127] 
Proc. of SPIE Vol. $9164916401-8$

Downloaded From: https://www.spiedigitallibrary.org/conference-proceedings-of-spie on 26 Apr 2023 Terms of Use: https://www.spiedigitallibrary.org/terms-of-use 


\section{Authors}

Numbers in the index correspond to the last two digits of the six-digit citation identifier (CID) article numbering system used in Proceedings of SPIE. The first four digits reflect the volume number. Base 36 numbering is employed for the last two digits and indicates the order of articles within the volume. Numbers start with 00, 01, 02, 03, 04, 05, 06, 07, 08, 09, OA, OB...0Z, followed by 10-1Z, 20-2Z, etc.

Aas, Mehdi, 34

Al-Balushi, Ahmed A., 18

Alexandrou, Antigoni, 15

Altman, David, 35

Anderson, Dana, 07

Andres-Arroyo, Ana, 11, 1L

Angelsky, Oleg $\vee ., 2 \mathrm{~N}$

Angstmann, C., $1 \mathrm{~L}$

Antalik, Marian, $2 \mathrm{P}$

Arita, Yoshihiko, OK

Arroyo-Carrasco, Maximino Luis, 2V

Arzola, Alejandro V., OL

Aumann, Andreas, 33

Banerjee, Ayan, 0G, 0M, 14

Barker, P. F., 03, 04

Barnett, S. M., OJ

Beltran-Perez, G., 3C

Bera, Sudipta K., 14

Bernatova, Silvie, 2P

Berns, Michael W., 13, 21

Besaga, Vira R., 2N

Bhuiya, Abdul M., 1M

Box, Stuart, 1Q

Brambila-Tamayo, Emma C., 39

Brodie, Graham W., 21

Bruot, Nicolas, 1Q

Brzobohatý, Oto, 2L, 36

Bui, Ann A. M., 13, 21

Burgin, Julien, $1 \mathrm{H}$

Cai, Chen, IW

Català, Frederic, OA

Cerecedo Nuñez, H. H., $2 U$

Chen, Hao, $1 \mathrm{M}$

Chen, Mingzhou, OK

Chen, Yue, 26

Chiou, Pei-Yu, 26

Chung, Aram, 26

Chvátal, Lukáš, OL, 2L

Cibula, Matthew A., 2T

Cicuta, Pietro, 1Q

Clark, Alasdair W., 25

Cochran, Sandy, 21

Cojoc, Dan, OS

Colas des Francs, Gérard, 30

Coleman, Victoria, 11

Cooper, Jonathan M., 25

Corsetti, S., 2W

Cotterell, Michael I., IY

Cruz, Gladys, 2I
Daniel, Rebekah, 35

Dantelle, Géraldine, 30

Debono, Luke, $1 Q$

Decombe, Jean-Baptiste, 30

Delville, Jean-Pierre, $1 \mathrm{H}, 2 \mathrm{C}$

Delville, Marie-Hélène, $1 \mathrm{H}$

Demore, Christine E. M., 21

Dholakia, Kishan, OK

Di Carlo, Dino, 26

Ding, Qing, $1 \mathrm{M}$

Doti, Rafael, $1 \mathrm{E}$

Duggan, Janet, 07

Dutta Gupta, Subhasish, OM

Ecoffet, C., 2M

Farkas, Daniel, 07

Farré, Arnau, OA, OU, 12

Faubert, Jocelyn, 1E

Fick, Jochen, 30

Fiszman, Nicolas, 15

Flores-Flores, E., 3C

Fonseca, P. G. Z., 03

Frawley, Mary C., 2X

Fu, Jinxin, 1V

Fury, C., $2 Z$

Gacoin, Thierry, 30

Ganchevskaya, Sofia V., 3D

Gao, Q., $1 \mathrm{~L}$

Ghadiri, R., 2F

Ghosh, Nirmalya, OM

Gibson, Graham M., 0J, 10

Gibson, Lachlan, 20

Gigan, Sylvain, Ol, 2S

Gordon, Reuven, 18

Guerreiro, A., 2M

Gurevich, E. L., 2F

Gusachenko, Ivan, 2X, 2Y

Hanna, Simon, 1Q

Hay, Rebecca F., 10

Henry, B., IL

Hernández Zavala, J. E., $2 U$

Hernández-Cordero, Juan, 29

Huang, Chen-Bin, 3B

Huang, Jer-Shing, 1G, 3B

Huant, Serge, 30

Hung, Chia-Chun, $1 \mathrm{G}$

Ivory, Megan, 07

Izaki, Kuniyoshi, 00

Jagadish, C., $1 \mathrm{~L}$

Jákl, Petr, OL, 2P, 36 
Ježek, Jan, 34, 36

Jones, P. H., $2 Z$

Jones, Stephanie H., 1X

Jorge, P. A. S., $2 M$

Kane, Bruce E., 05

Kaňka, Jan, 2L, 2P

Kellay, Hamid, 2C

Kemp, Scott, 11

Kesa, Peter, 2P

Khatibzadeh, Nima, 13, 2

Kiefer, J., 2W

Kimura, Yasuyuki, 00, 1P

King, Martin D., $1 \mathrm{X}$

Kiraz, Alper, 34

Köhler, Jannis, 2F, 33

Koll, Andrew T., 35

Kotar, Jurii, 1Q

Kotnala, Abhay, 18

Ksouri, Sarah Isabelle, 2F, 33

Kung, Yu-Chun, 26

Lavery, M. P. J., OJ

Lee, Michael P., OJ, 10

Le Gall, Antoine, 15

Li, Y. Lia, 04

Linnenberger, Anna, OY

Lokar, Žiga, 2B

López-Peña, Luis A., 29, 39

Lugo, J. E, 1E

MacDonald, Michael P., 21

Maksimyak, Andrew P., 2N

Maksimyak, Peter P., 2N

Mansuripur, Masud, $\mathrm{OB}$

Marsà, Ferran, OA, 12

Martín-Badosa, Estela, OA, OU

Mas, Josep, OU

Mason, Bernard J., IY

Masson, Jean-Baptiste, 15

Mattern, Manuel, 33

Mavrogordatos, T., 03

Mazilu, Michael, OK

McDermott, D., 20

McGloin, D., 2W

McIntyre, David H., 2T

Memoli, G., $2 Z$

Méndez, Guadalupe, 2V

Miles, Mervyn, $1 Q$

Miles, R. E. H., 2W

Millen, J., 03, 04

Mondal, Argha, OG, 14

Monteiro, T. S., 03

Montes-Usategui, Mario, OA, OU, 12

Morozov, Andrey A., 3D

Muševič, Igor, 2B

Nagornykh, Pavel, 05

Neale, Steven L., 25, 3C

Nic Chormaic, Síle, 2X, $2 Y$

Nieminen, Timo A., 13, 21, 20

Oddershede, Lene B., 11

Okubo, Shogo, IP

Oliva, A., 2M
Olson Reichhardt, C. J., 1N, 20

O'Mahoney, Paul, 21

Orr-Ewing, Andrew J., IY

Ostendorf, Andreas, 2F, 33

Osterman, Natan, 2B

Ott, Dino, 11

Ou-Yang, H. Daniel, 1V, 2L

Padgett, Miles J., OJ, 10

Padilla Sosa, P., $2 \mathrm{U}$

Páez López, Rafael, 2V

Perez, Maximillian A., 07

Perronet, Karen, 15

Peterka, Darcy S., OY

Petit, Julien, 2C

Phillips, David B., 0J, 10, 1Q

Pilát, Zdeněk, 34

Piwonka, I. O., $1 \mathrm{~L}$

Porfirev, Alexey P., 3A, 3D

Power, Rory M., IW

Preece, Daryl, 20

Preston, Thomas C., IY

Queirós, R. B., 2M

Quirin, Sean, OY

Ramirez-San-Juan, J. C., 3C

Ramos-García, Ruben, 2V, 3C

Ray, D., IN

Reboud, Julien, 25

Reece, Peter, J., 1I, 1L

Reichhardt, C., IN, 20

Reid, Jonathan P., 1W, 1Y, 2W

Reihani, S. Nader S., 11

Richly, Maximilian U., 15

Rivière, David, 2C

Robert de Saint Vincent, Matthieu, 2C

Rocha, Yesenia, 2

Rodrigues Ribeiro, R. S., $2 M$

Roxworthy, Brian J., $1 M$

Roy, Basudev, OG, OM, 14

Roy, Soumyajit, OG

Rubinsztein-Dunlop, Halina, 13, 21, 20

Rykov, Mikhail A., 3D

Salazar-Romero, Yadira, 29

Salim, Evan, 07

Sanchez, Noemi, $1 \mathrm{E}$

Sancho-Parramon, Jordi, OU

Sergides, Marios, $2 Y$

Šerý, Mojmír, OL, 2P, 34

Sheng, Yunlong, $O R$

Shibata, Shuhei, IP

Si, Satyabrata, $1 \mathrm{H}$

Siler, Martin, 36

Simpson, Stephen H., 0J, 1Q, IW

Škarabot, Miha, 2B

Skidanov, Roman V., 3A, 3D

Soppera, O., $2 M$

Spalding, Gabriel C., 21

Speirits, F. C., OJ

Stilgoe, Alexander B., 13, 21

Tan, H. H., $1 \mathrm{~L}$

Teitell, Michael A., 26 
Terborg, Roland A., 29, 39

Toe, Wen Jun, 11, 1L

Tomori, Zoltan, $2 \mathrm{P}$

Torres, Juan P., 29, 39

Torres-Hurtado, S. A., 3C

Toussaint, Kimani C., Jr., IM

Truong, Viet Giang, 2X, $2 Y$

Tsai, Wei-Yi, 3B

Türkcan, Silvan, 15

Valdivia-Valero, Francisco J., 30

Velasco-Gutiérrez, Cristian R., 39

Vigueras Zuñiga, M. O., $2 U$

Volke-Sepúlveda, K., 29, 39

Volpe, Giorgio, 0l, $2 S$

Volpe, Giovanni, 0l, $2 S$

Wang, Fan, 11

Wang, Han, 2

Ward, Andrew D., $1 \mathrm{X}$

Westbrook, Nathalie, 15

Witte, Christian, 25

Wright, Ewan M., OK

Wu, Ting-Hsiang, 26

Yang, Ya-Tang, $1 \mathrm{G}$

Yu, Lingyao, OR

Yuste, Rafael, OY

Zemánek, Pavel, OL, 2L, 2P, 34, 36

Zhang, Shu, 20

Zyla, Gordon, 33

Proc. of SPIE Vol. $9164916401-11$ 
Proc. of SPIE Vol. $9164916401-12$

Downloaded From: https://www.spiedigitallibrary.org/conference-proceedings-of-spie on 26 Apr 2023 Terms of Use: https://www.spiedigitallibrary.org/terms-of-use 


\title{
Conference Committee
}

\author{
Symposium Chairs
}

Satoshi Kawata, Osaka University (Japan)

Manijeh Razeghi, Northwestern University (United States)

Symposium Co-chairs

David L. Andrews, University of East Anglia Norwich (United Kingdom) James G. Grote, Air Force Research Laboratories (United States)

Conference Chairs

Kishan Dholakia, University of St. Andrews (United Kingdom)

Gabriel C. Spalding, Illinois Wesleyan University (United States)

\section{Session Chairs}

1 Toward (Or In) the Quantum Limit of Opto-Mechanics

Michael Mazilu, University of St. Andrews (United Kingdom)

2 Foundations of the Electromagnetic Theory of Force and Momentum Gabriel C. Spalding, Illinois Wesleyan University (United States)

3 Beam Shaping and Aberration/Wavefront Correction

Gabriel C. Spalding, Illinois Wesleyan University (United States)

4 Helicity and Optical Angular Momentum

Kishan Dholakia, University of St. Andrews (United Kingdom)

5 Optically Driven Microrheology and Mechanical Properties

Jean-Pierre Delville, Université Bordeaux 1 (France)

6 Using the Photonic Toolbox to Study Cells and Their Organelles

Michael W. Berns, University of California, San Diego (United States)

7 Enhanced Sensitivity and Resolution of Optical Force Actuators

Giovanni S. Volpe, Bilkent University (Turkey)

$8 \quad$ Single-Molecule Manipulation and Study

Kishan Dholakia, University of St. Andrews (United Kingdom)

9 Alternative Methods

Hiroshi Masuhara, National Chiao Tung University (Taiwan) 
10 Photonic Devices for Optically Induced Forces

Masud Mansuripur, College of Optical Sciences, The University of Arizona (United States)

11 Near-Field Micromanipulation, Plasmonic, and Nanoparticle Trapping

Romuald Houdré, Ecole Polytechnique Fédérale de Lausanne (Switzerland)

12 Studies of Active Swimmers/Hydrodynamics

Vernita Gordon, The University of Texas at Austin (United States)

13 Statistical Mechanics of Small Systems

Roberto Di Leonardo, Università degli Studi di Roma La Sapienza (Italy)

14 Optical Manipulation of Matter through Gaseous Media Kishan Dholakia, University of St. Andrews (United Kingdom)

15 Optical Sorting, Optical Lab-on-a-Chip, and Microfluidics Simon Hanna, University of Bristol (United Kingdom)

16 Optically Bound Matter

Gabriel C. Spalding, Illinois Wesleyan University (United States)

17 Optofluidics and Optically Shaped Structures

Etienne Brasselet, Université Bordeaux 1 (France)

18 Optically Manipulated Robotics and Novel Samples

Kishan Dholakia, University of St. Andrews (United Kingdom)

Gabriel C. Spalding, Illinois Wesleyan University (United States)

19 In Memory of Dmitri Petrov

Danut-Adrian Cojoc, Laboratorio Nazionale TASC (Italy)

20 The Final Session

Karen P. Volke-Sepúlveda, Universidad Nacional Autónoma de México (Mexico) 